\title{
STICHWORT
}

\section{Religiöses und Spirituelles Coping}

Monika Lang

"Coping“ ist nach Snyder \& Pulver (vgl. ebd., In: Snyder, 2001) eines der zentralen psychologischen Konzepte der Gegenwart, dass sie folgendermaßen definieren: „[...] coping reflects thinking, feeling, or acting so as to preserve a satisfied psychological state when it is threatened" (ebd.,4). Bei diesem Verständnis von Coping geht es weniger um den erfolgreichen Umgang mit alltäglichen Anforderungssituationen, sondern um die Auseinandersetzung mit existenziellen Krisen und belastendend Lebenssituationen- und ereignissen, um die „big events“ im Leben eines Menschen, die die bisherige Lebensgestaltung infrage stellen und die psychische Gesundheit gefährden können. Bereits in den 6oer und 7oer Jahren stellte die Stressforschung individuelle Unterschiede in der Reaktion auf belastende Ereignisse fest. Vor allem die persönliche Konfrontation mit Krankheit, Behinderung oder Tod löst Stress und in der Folge verschiedene emotionsregulierende und problemlösende Anstrengungen aus, die „subjektiv wahrgenommene Diskrepanz zwischen Bewältigungsfähigkeit und Umweltanforderung" (Hobfoll \& Buchwald 2004, 11) wieder zu schließen. Dieses eher homöostatische Modell der Adaptation bei Stress erweiterte sich u.a. im „Cognitive Approaches“Ansatz von Lazarus \& Folkman (1984) zu einem transaktionalen Verständnis von Coping. Nach Maynard, Gorsuch \& Bjorck $(2001,65)$ wird Coping hier verstanden ,as ongoing transactional process between the person and his or her environment, a process impacted by both cognitive appraisal and coping behaviour". Das Individuum analysiert demnach jeweils seine Person-Umwelt-Beziehungen und schätzt auf diesem Hintergrund die Bedeutsamkeit der je- weiligen Situation ein. Dabei wird zwischen Irrelevanz, Bedrohung, Verlust/Schaden, Herausforderung und Gewinn unterschieden. In einem zweiten Bewertungsschritt werden die persönlich zugänglichen Ressourcen und Bewältigungsstrategien in ihrem Verhältnis zur Anforderungssituation eingeschätzt und ein entsprechendes Copingverhalten wird gewählt. Nach Carver, Weintraub \& Scheier (1989) hat bereits Lazarus diese Prozesse nicht als einen rein linearen Ablauf beschrieben, sondern mögliche zirkuläre Rückkopplungsprozesse betont. Wenn sich z. B. eine gewählte Bewältigungsstrategie als nicht effektiv erweist, so kann dies zur Folge haben, dass sich die Einschätzung der Anforderungssituation verändert und der Betroffene diese nicht mehr als Herausforderung, sondern eher als Bedrohung wahrnimmt. Die Frage nach dem Einfluss situativer (z.B. der jeweiligen sozialen Rolle) und personaler Faktoren (z.B. der Annahme eher stabiler Persönlichkeitsdimensionen und Wahrnehmungsstile) wird dabei gegenwärtig sehr unterschiedlich beantwortet. Ebenso wird die mangelnde Berücksichtigung kultur- und geschlechtsspezifischer Copingmuster und die überzogene Orientierung an der westlichen Kultur mit ihrer „dominierenden Kontroll- und Kompetenzideologie" kritisiert (vgl. Hobfoll \& Buchwald 2004, 16).

Die Möglichkeit der Persönlichkeitsentwicklung durch Coping wird von Pargament betont: „Through coping, resources are not only used, they are developed; burdens are not only taken on, they are lightened" (ebd., 1997, 104). Er versteht „Coping“ als „[...] search for the significance in time of stress" (ebd., 1997, 90) 
und zitiert W. James, der die Frage nach dem Sinn bzw. der Bedeutung einer Lebenssituation, als "the hot place in a man's consciousness" bezeichnet hat $(1902,193)$. James ist auch der erste Wissenschaftler, der ein Interesse an der systematischen Erforschung von Psychologie und Religion entwickelt, später gefolgt von C. G. Jung (1933) und G. Allport (1950). Im Rahmen der Mental-Health-Forschung wurde in den vergangenen Jahrzehnten ebenfalls häufig nach den positiven und negativen Einflüssen eines religiösen Glaubens auf die geistige bzw. seelische Gesundheit und das emotionale Wohlbefinden gefragt und überwiegend ein schwach positiver Zusammenhang festgestellt (vgl. u.a. Koenig \& Larson 2001). Koenig \& Larson benennen drei Aspekte, als mögliche Erklärung für die salutogenen Wirkungen eines religiösen Glaubens: Die Unterstützung eines optimistischen Weltbildes und Deutungsrahmens, die Betonung von Werten wie Vergebung, Mitgefühl und Hilfsbereitschaft sowie die Stärkung der familiären Beziehungen und eines tragfähigen sozialen Netzwerkes (vgl. ebd., 2001, 72). Deutlich seltener finden sich empirische Belege für negative und belastende Auswirkungen eines religiösen Glaubens, die sich aber vor allem in einer negativen Beziehung zwischen einer extrinsischen, eher oberflächlichen und nutzenorientierten religiösen Orientierung und psychischer Gesundheit abbilden (vgl. Grom 2000).

Die Verknüpfung der stresstheoretisch orientierten Copingforschung mit der Frage nach dem Stellenwert und Einfluss eines religiösen Glaubens auf die Bewältigung von Krisensituationen hat sich in den letzten Jahrzehnten zu einem eigenen internationalen Forschungsfeld „Religious/Spiritual Coping“ entwickelt. In seinem Grundlagenwerk „The Psychology of Religion and Coping“ (1997) beschäftigt sich der Religionspsychologe K.I. Pargament mit der Frage „[...] when, why, and how religion comes to life" (ebd., 68). Besonders in Situationen, in denen die Gesundheit bedroht und die physische oder psychische Funktionsfähigkeit eingeschränkt ist, scheint religiöser Glaube an Bedeutung zu gewinnen. Siegel \& Schrimshaw (2002) begründen dies vor allem mit der angstauslösenden Erfahrung der Begrenztheit der persönlichen Kontroll- und Einflussmöglichkeiten. Einzelne Aspekte einer belastenden Situation können zwar durch problemlöseorientiertes Copingverhalten verändert werden, der Bedrohungskern, z.B. eine Krebserkrankung oder eine körperliche Beeinträchtigung nach einem Unfall, bleibt aber bestehen und kann dann effektiver durch emotionsregulierende Strategien bewältigt werden. Vor allem Personen, die ihr religiöses oder spirituelles Orientierungssystem bereits vor dem kritischen Lebensereignis stärker ausgebildet haben, beziehen sich dann in der Krise auch stärker darauf und scheinen dann bei den positiven Copingeffekten auch eher zu „profitieren" (vgl. Pargament \& Brant 1998, 123).

Die scheinbar einfache Frage, ob religiösem Glauben im Rahmen eines Bewältigungsprozesses eine hilfreiche, belastende oder irrelevante Funktion zukommt, kann nach Pargament (1997) daher in dreifacher und verwirrender Weise jeweils bejaht werden, da Religion ein facetten-und formenreiches Feld darstellt und daher auch Religious/Spiritual Coping „multipurpose“ und „multiform“ ist (vgl. ebd., 118).

Mit Bezug zu folgenden Veröffentlichungen der Autorin:

Lang, M. (1999). Geistige Behinderung - Bewältigung und Religiöser Glaube. Eine Interviewstudie mit Müttern von Jugendlichen und Erwachsenen mit einer geistigen Behinderung. Frankfurt a. M.: Peter Lang, Reihe VI Psychologie, Bd. 640

Lang, M. (2013, in Druckvorb.). „Das andere Glück" - Bewältigungs- und Transformationsprozesse bei Müttern und Vätern von Kindern mit Behinderung unter besonderer Berücksichtigung von Religious Coping. Habilitationsschrift, Philipps-Universität Marburg. 